\title{
Retos y aprendizajes de integrar psicología positiva y ejercicio físico en los Estudios Generales:
} relatos desde la praxis académica

Challenges and Learning from Integrating Positive Psychology and Physical Exercise in the General Studies Department: Stories from Academic Praxis

\section{Kristy Barrantes-Brais ${ }^{1}$ \\ Universidad Nacional}

Costa Rica

\section{Marcela Gutiérrez Miranda \\ Universidad Nacional \\ Costa Rica}

Maribel León Fernández ${ }^{3}$

Universidad Nacional

Costa Rica

María de los Ángeles Sancho Ugalde

Universidad Nacional

Costa Rica

1 Doctora en Ciencias del Movimiento Humano, Máster en Salud Integral y Movimiento Humano. Acadêmica y psicóloga de la Universidad Nacional de Costa Rica. Correo eletrónico: kristy.barrantes.brais@una.cr

2 Maestría en Promoción de la Salud y Desarrollo Social. Médica, académica e investigadora de la Universidad Nacional de Costa Rica.

Correo electrónico: marcela.gutierrez.miranda@una.cr

3 Doctora en Educación, Magister Scientiae en Gerontología Académica del Centro de Estudios Generales de la Universidad Nacional. Correo eletrónico: maribel.leon.fernandez@una.cr

4 Maestría en Lingüística, Licenciada en la Enseñanza del Español. Académica del Centro de Estudios Generales en Universidad Nacional de Costa Rica.. Correo electrónico: maria.sancho.ugalde@una.cr 


\title{
Resumen
}

El presente artículo se desarrolla como un relato de experiencia que resulta de un proceso académico impartido en la Universidad Nacional, específicamente en el Centro de Estudios Generales. El proceso tuvo como objetivo promover el bienestar psicológico de estudiantes universitarios mediante intervenciones en psicología positiva (IPP) y ejercicio físico y, a su vez, desarrollar capacidades en el personal académico del Centro de Estudios Generales para gestionar intervenciones de psicología positiva. La metodología empleada en este proyecto académico consistió en una serie de intervenciones mediante los siguientes tres cursos: 1) ambiente y salud, 2) la imagen de la persona adulta mayor en los diferentes textos y 3) taller de expresión literaria: cuento y poesía. Las experiencias particulares del desarrollo de las intervenciones en cada curso se reseñan en todo este relato. Asimismo, de lo anterior se obtuvieron los retos y aprendizajes para cada curso; además, se brindó capacitación sobre las bases teóricas y el procedimiento de implementación de las tres IPP reseñadas. Al finalizar el proceso de capacitación, de manera conjunta se determinaron las formas pertinentes de incluir los principios e intervenciones de psicología positiva y educación positiva en la estrategia metodológica del programa de curso. Dicha experiencia generó posibilidades para que las diferentes disciplinas trabajaran de manera estrecha en un plan común para la praxis académica en la docencia e investigación; como pedagogía multidisciplinaria se enrumba hacia la interdisciplinariedad.

Palabras clave: bienestar estudiantil; humanidades; interdisciplinariedad; academia.

\begin{abstract}
This article is written as an account of experience resulting from an academic process conducted at the National University of Costa Rica, specifically at the Center for General Studies. The process aimed to promote the psychological well-being of university students through interventions in positive psychology (IPP) and physical exercise and, in turn, to develop skills in the academic staff of the Center for General Studies to manage positive psychology interventions. The methodology used in this academic project consisted of a series of interventions through the following three courses: 1) environment and health, 2) the image of the elderly person in the different texts and 3) literary expression workshop: story and poetry. The particular experiences of the interventions conducted in each course are outlined throughout this account. Likewise, challenges and learning for each course were obtained from these interventions. Training was provided on theoretical bases and the implementation procedure of the three IPPs reviewed. At the end of the training process, the relevant ways of including the principles and interventions of positive psychology and positive education in the methodological strategy of the course program were jointly determined. This experience generated possibilities for the different disciplines to work closely on a common plan for academic praxis in teaching and research; as a multidisciplinary pedagogy, it is rooted in interdisciplinarity.
\end{abstract}


Keywords: student well-being; humanities; interdisciplinarity; academia.

\section{Introducción y contextualización}

Este relato refleja el proceso de gestión y ejecución para promover el bienestar psicológico de estudiantes universitarios, mediante intervenciones en psicología positiva y ejercicio físico. La experiencia permitió, además, desarrollar capacidades en el personal académico del Centro de Estudios Generales (CEG) de la Universidad Nacional (UNA), para gestionar intervenciones de psicología positiva. Este ejercicio académico formó parte de uno de los componentes de un proceso de tesis en el Doctorado en Ciencias del Movimiento Humano, programa conjunto de la Universidad de Costa Rica y la UNA (Barrantes, 2017). La experiencia profesional de las autoras en diversas áreas del conocimiento y en contacto constante con estudiantes propició la formulación de una idea de investigación centrada en promover el bienestar de la población estudiantil, de la mano con la labor docente en los cursos de humanidades.

Es en este contexto que, en el II ciclo lectivo del 2015, se llevó a cabo en el CEG una serie de intervenciones, las cuales formaron parte de tres cursos: Taller de expresión literaria: cuento y poesía, La imagen de la persona adulta mayor en los diferentes textos y Ambiente y salud. Las experiencias particulares del desarrollo de las intervenciones en cada curso se reseñan más adelante y constituyen el eje central de este relato. Resulta fundamental señalar que el componente de psicología positiva fue desarrollado por las docentes, luego de un periodo de sensibilización y formación activa facilitado por una profesional en psicología. El componente de ejercicio físico fue proporcionado por un profesional en educación física.

El enfoque de la psicología positiva empieza a tomar forma a finales de la década de 1990, como una respuesta a la "forma tradicional" de hacer psicología (centrada en los trastornos y las carencias), y propone volver la mirada a aquellos elementos que "hacen que la vida merezca ser vivida" (Seligman y Csikszentmihalyi, 2000, p. 5), de manera que se favorezca el funcionamiento humano óptimo (que las personas puedan ser y dar lo mejor de sí mismas) (Park y Peterson, 2009a). Se ha posicionado como el estudio científico de fortalezas, rasgos, motivaciones, virtudes y conductas humanas positivas, con el propósito de comprender y optimizar estas cualidades en la vida de las personas; de contribuir a una comprensión científica más completa y equilibrada de la experiencia humana, así como de transmitir lecciones valiosas acerca de cómo construir una vida feliz, saludable, productiva y significativa (Park y Peterson, 2009a; Seligman Steen, 
Park, y Peterson, 2005; Sheldon y King, 2001). Desde esta perspectiva, no se niega la existencia de dificultades y eventos adversos, pero se evita centrar la atención en estos y, más bien, se propone un balance que permita reconocer las potencialidades y recursos positivos en las personas, grupos e instituciones.

Las intervenciones de psicología positiva (IPP) se han definido como aquellas estrategias psicológicas cuyo objetivo es generar o incrementar emociones, conductas y cogniciones positivas - por lo tanto, aumentar el bienestar de los individuos o grupos- (Parks y Biswas-Diener, 2013; Sin y Lyubomirsky, 2009). Se basan en el conocimiento acumulado sobre los procesos afectivos, conductuales, cognitivos y motivacionales que posibilitan el bienestar (Schueller, Kashdan y Parks, 2014). En este proceso, se utilizaron las tres IPP sobre las cuales existe más evidencia en cuanto a su impacto positivo en indicadores de bienestar psicológico: el reconocimiento y uso de fortalezas personales, los diarios de gratitud y "mi mejor yo posible" (Bolier et al., 2013; Hervás et al., 2008; Hone, Jarden y Schofield, 2014; Sin y Lyubomirsky, 2009). Se detallan, a continuación, los principales componentes teóricos de cada intervención y se describe la metodología de su aplicación en los cursos del CEG.

Las fortalezas personales son rasgos positivos que se reflejan en pensamientos, sentimientos y conductas, así como posibilitan el funcionamiento y desempeño óptimo de las personas (Park, Peterson y Seligman, 2004). Constituyen características positivas que existen en diferentes grados en las personas, como en un continuum, y pueden ser "cultivadas" o desarrolladas de forma deliberada (Park y Peterson, 2009b). La identificación (reconocimiento) y uso (puesta en práctica de forma consciente) de fortalezas personales fue una de las primeras intervenciones documentadas por los pioneros de la psicología positiva (Seligman et al., 2005). Mediante cuestionarios o ejercicios reflexivos a partir del listado de 24 fortalezas, las personas identifican sus principales áreas fuertes y se les solicita que reconozcan cómo hacen o han hecho uso de ellas; posteriormente, se les anima a emplearlas en nuevas formas, en su vida cotidiana (Peterson, 2006; Seligman, Rashid y Parks, 2006).

El ejercicio de la gratitud constituye un predictor de satisfacción con la vida y se asocia con indicadores de bienestar como la capacidad de disfrutar experiencias placenteras y el sentido de vida (Peterson, Ruch, Beermann, Park y Seligman, 2007). La intervención denominada "diario de gratitud" consiste en anotar, al final de cada día (durante una o varias semanas), al menos tres cosas que la persona valora que salieron bien o fueron agradables/beneficiosas y por las cuales se siente agradecida (Emmons y McCullough, 2003; Wood, Froh y Geraghty, 2010). 
Por su parte, "mi mejor yo posible" se clasifica como una de las intervenciones basadas en la proyección a futuro y la promoción del optimismo; es coherente con las líneas de investigación e intervención desde la psicología positiva (Schueller et al., 2014). En esta intervención, se le indica a la persona que se visualice a sí misma a futuro, habiendo conseguido sus metas y alcanzado todo su potencial. Se solicita que escriba los detalles de lo que observa en ese futuro, así como las fortalezas personales que considera necesarias para conseguir lo deseado; la duración y frecuencia del ejercicio es variable (King, 2001; Layous, Nelson y Lyubomirsky, 2013; Sheldon y Lyubomirsky, 2006).

Las intervenciones basadas en el ejercicio físico pueden enmarcarse en una amplia gama de iniciativas, estrategias y programas sistemáticos centrados en la práctica y promoción de la actividad física (Bauman et al., 2008). Estas intervenciones pueden considerar uno o más de los siguientes aspectos: pretenden incidir en los hábitos de las personas en diversos espacios (lugares de estudio o trabajo, actividades domésticas, ámbitos recreativos, vida en comunidad); pueden integrar estrategias educativas para el desarrollo de habilidades, así como modificaciones en los espacios físicos y sociales; privilegian un enfoque en la práctica de ejercicio como parte de un estilo de vida activo y saludable; promueven la integración social y el disfrute (Bauman et al., 2008; Hoehner et al., 2008; Huijg et al., 2015; Laine et al., 2014). A partir de lo referido por experiencias previas, se diseñó un protocolo para que el estudiantado de dos de los cursos realizara ejercicio físico a intensidades de baja a moderada, durante 45 minutos semanales.

Tomando en consideración los potenciales beneficios de las intervenciones reseñadas, se propuso trabajar con población estudiantil del CEG de la UNA, en el marco de la promoción de la salud y el bienestar, considerada una labor en las instituciones de educación superior (Miller y Chung, 2009).

El CEG promueve, en su labor sustantiva, la incorporación de un nuevo humanismo que representa una perspectiva ético-filosófica referida a la naturaleza humana actual (Baraona, 2013) y que reconoce las necesidades del hombre moderno. Esta perspectiva toma en consideración la compleja realidad en la que se encuentra inmersa la raza humana del siglo XXI y propone una nueva ética de la vida social, con la cual se gesta una forma de generar y transmitir conocimiento incluyendo una visión holística de la realidad que prioriza el bienestar del individuo. Desde tal noción, se sugiere reflexionar sobre el bienestar como producto de los procesos de formación y desarrollo que respetan al individuo en sus particularidades, el entorno y las complejas interacciones establecidas. Es este nuevo humanismo que guía la labor del CEG, el que facilitó el desarrollo de una propuesta innovadora 
que responde a las necesidades de los estudiantes y contribuye con su desenvolvimiento integral (Baraona, 2013; Baraona y Mata, 2015; Baraona y Mora, 2017).

En esta propuesta, se consideró fundamental que las intervenciones se implementaran en el entorno cotidiano de la población estudiantil y que se pudieran incorporar como parte de sus vivencias también cotidianas en el aula. Existe reconocimiento de que en estudios que pretenden demostrar el efecto de algún tipo de intervención resulta recomendable que esta no sea dirigida por los investigadores, sino por personas (apropiadamente capacitadas) que estén familiarizadas con el contexto y se desenvuelvan en él de forma natural (Flay et al., 2005; Hone et al., 2014). Es en esta línea que se propuso generar espacios para la creación y fortalecimiento de capacidades en el personal docente, con el fin de que las IPP fueran implementadas por las profesoras en sus clases.

En el semestre previo a la implementación de las intervenciones, se desarrolló un proceso de sensibilización y formación activa con metodología participativa. Durante cuatro sesiones de dos horas (con asignaciones entre sesiones), la profesional en psicología y las académicas trabajaron en espacios para la revisión, comprensión e interiorización de conceptos y principios de psicología positiva y educación positiva aplicables a la mediación pedagógica de los cursos de Estudios Generales. Se brindó capacitación con respecto a las bases teóricas y el procedimiento para implementar las tres IPP reseñadas. Al finalizar el proceso de capacitación, de manera conjunta se determinaron las formas pertinentes de incluir los principios e intervenciones de psicología positiva y educación positiva en la estrategia metodológica del programa de los cursos, de manera que se entrelazaran con los contenidos y objetivos de cada uno de ellos. Se definieron los contenidos por trabajar en las intervenciones de psicología positiva, durante seis sesiones semanales de 45 minutos cada una (parte de la clase regular de cada curso), y se desarrolló un protocolo para facilitar intervenciones, con el detalle de cada sesión y las asignaciones correspondientes.

Durante el proceso de implementación de las intervenciones, cada docente sostuvo reuniones de realimentación con la psicóloga, después de cada sesión, lo cual facilitó la discusión constante sobre aspectos teóricos y metodológicos por retomar en las siguientes sesiones, en un esfuerzo interdisciplinario para promover el bienestar del estudiantado.

De esta manera, la experiencia desde cada curso tuvo características y resultados muy diferentes, pero, desde la psicología positiva, quedó demostrado que esta permitió entrelazar los contenidos de cada uno, teniendo como fin último el bienestar de la población estudiantil. A continuación, se explican las tres experiencias, 
con base en los retos y aprendizajes desde los cursos Taller de expresión literaria: cuento y poesía, La imagen de la persona adulta mayor en los diferentes textos y Ambiente y salud.

Se consideró que estos cursos permitirían una integración provechosa de los componentes de psicología positiva y ejercicio físico con los componentes curriculares, de forma que se facilitarán procesos de desarrollo personal, profundizando así en la puesta en práctica de la visión humanística del estudiantado (como seres humanos integrales).

\section{Retos y aprendizajes desde el curso Taller de expresión literaria: cuento y poesía}

Con base en las IPP denominadas "identificación y uso de fortalezas personales", "mi mejor yo posible" y "diario de gratitud", los estudiantes universitarios del curso Taller de expresión literaria: cuento y poesía lograron el objetivo general del curso, el cual consiste en promover el acercamiento a la escritura, mediante el desarrollo de hábitos lectores y la discusión de modelos estético-literarios. Con dichas intervenciones, se interrelacionan la psicología con la literatura. En las creaciones literarias, los jóvenes escribieron sobre fortalezas y la mejor versión de personajes literarios, también gestaron personajes agradecidos, con base en su diario de agradecimiento, lo que les permite conocerse más a sí mismos, a sus compañeros y a la profesora del curso.

En el Taller de expresión literaria: cuento y poesía, se presenta como un reto la búsqueda constante de potencialidades positivas en los educandos, cuando cada uno de ellos expone su propia interpretación literaria, la cual corresponde a una idea particular de mundo. De tal modo, los alumnos entran en contacto con la realidad disímil o semejante de los compañeros, factor que fortalece la identidad, la diversidad de grupo y la pluralidad de percepciones tanto líricas como narrativas.

Para cumplir con lo antes mencionado, en el II ciclo 2015, durante seis semanas, se realizaron actividades de psicología positiva. Con base en los trabajos relacionados con el reconocimiento y uso de fortalezas, así como en "mi mejor yo posible", los estudiantes destacaron los aprendizajes presentados a continuación:

- Visualizar su participación en las comunidades donde viven y en la misma Universidad Nacional.

- $\quad$ Reconocer que sus fortalezas personales les protegen ante adversidades.

- Crear soluciones ante los retos que se les presentan en la vida.

- Ayudar a otros, acercarse a personas que necesitan apoyo. 
- $\quad$ Promover cambios en sus actividades cotidianas, por ejemplo, participar en eventos que sean de su agrado.

- Aplicar sus fortalezas personales en el futuro, cuando se desempeñen como profesionales.

Respecto al diario de gratitud, esta actividad les ayudó a prestar más atención a los detalles de la cotidianidad y a estar más agradecidos con lo bueno y positivo que les sucede en su vida, en vez de estar renegando por situaciones no tan positivas.

Otro reto importante fue el cambio en la metodología del curso, al integrar la psicología positiva con la literatura; por ejemplo, en el curso se ve la película "Il Postino", basada en la vida del poeta chileno Pablo Neruda. Para realizar dicha integración, se solicitó a los estudiantes, escoger dos personajes de la película y describir sus fortalezas; estas fueron dos de las respuestas:

El poeta Neruda tiene el don de la sencillez y las ganas de ayudar a las personas; por eso su primera fortaleza sería Amabilidad y generosidad y lo demuestra cuando ayuda a Mario a entender la poesía. También, la Perspectiva (sabiduría) lo caracteriza porque Mario lo visualiza como el maestro. Aprecio de la belleza y la excelencia, aprovecha esta fortaleza a la hora de escribir sus poemas donde resalta y compara las magnificencias de la naturaleza. Posee la fortaleza de Esperanza, optimismo y visión del futuro porque se esfuerza por ganar el premio de literatura. Por último, posee el don de la paciencia y la perseverancia. Si pudiera adoptar el papel de alguno de los personajes, elegiría ser Pablo Neruda porque tengo paciencia para enseñar y me gusta compartir con los demás lo que sé y he aprendido en la vida. Amo la poesía y las metáforas. (Comunicación de una estudiante del curso Taller de expresión literaria).

Mario Roúpolo refleja a lo largo de toda la película, la fortaleza del Deseo de aprender sobre poesía y sobre la forma de pensar del poeta. La Gratitud también lo caracteriza, además de la Tenacidad, diligencia y perseverancia; no deja de esforzarse por aprender y tampoco renuncia a su amor por Beatrice. Su condición resalta su Modestia y humildad. Cuando se casa con Beatrice Russo demuestra que posee la capacidad de amar y ser amado. Además, todo en él se manifiesta la curiosidad en persona. (Comunicación de una estudiante del curso Taller de expresión literaria). 


\section{Retos y aprendizajes desde el curso La imagen de la persona adulta mayor en los diferentes textos}

En el curso La imagen de la persona adulta mayor en los diferentes textos, la ruptura de mitos y estereotipos sobre el envejecimiento y la vejez, así como la promoción de estilos de vida saludable son parte fundamental. La incorporación de temas relacionados con la psicología positiva bajo la modalidad de intervenciones combinadas en las que se realizaba también ejercicio físico de intensidad baja a moderada, facilitada por un graduado en educación física, fue una experiencia interdisciplinaria de gran impacto.

Durante la clase, se dio una conjunción de saberes entre la psicología positiva, la educación física y la gerontología para la construcción de un envejecimiento y una vejez saludables. En su parte gerontológica, se hacía énfasis en que las personas, a lo largo de su proceso de envejecimiento, debían desarrollar sus distintas dimensiones para que este y la vejez sean activos y saludables. Con el propósito de ejemplificar esto, se estudiaron las zonas azules en las cuales las personas adultas mayores llegan a sobrepasar los 100 años de vida en condiciones de salud envidiables y en donde unos de los comunes denominadores son actividad física, tener metas, poseer un sentido de vida, sentirse todavía competentes y agradecidos por todo lo que han vivido y poder mantener una vida activa en su familia y su comunidad sintiéndose útiles, amados e importantes (Buettner, 2008).

Se facilitaron aprendizajes para comprender que, al llegar la vejez con sus ganancias y pérdidas, es de suma importancia identificar las fortalezas, con el fin de sumarlas a sus recursos para vivir mejor la vida y, al entrar en el proceso de gerotrascendencia, reconocer y practicar la gratitud con mayor frecuencia y fuerza. La cual es entendida como:

(...) proceso de cambio, de una visión más materialista de la vida a una visión más espiritual, permite al sujeto, por un lado elaborar ciertas pérdidas y por el otro crecer personalmente, ya que podrá dar lugar a la verdadera noción de experiencia o sabiduría de la vida. (Iacub, 2007, p. 3)

Así, desde la psicología positiva, la población estudiantil pudo identificar sus fortalezas y practicar la gratitud. Además, en el nivel personal, reconocieron la importancia, durante el envejecimiento, de satisfacer sus necesidades de autonomía, autoaceptación y autorrealización y, en el nivel interpersonal, comprendieron que las personas adultas mayores necesitan, al igual que ellos, establecer, mantener y fortalecer vínculos familiares, sociales y comunitarios, en los que se desarrollan diversos grados de apoyo social y reciprocidad afectiva. 
La población estudiantil pudo reconocer nuevos ámbitos de aplicación de sus fortalezas, como su carrera, su hogar y su comunidad; también lograron, gracias a ellas, principalmente la perseverancia y la creatividad, sacar adelante tareas que antes les resultaban poco agradables o complicadas. Debido al uso de sus fortalezas y poniendo en práctica sus habilidades, alcanzaron mejorar su estilo de vida y ayudar a otros, así como manifestar actos de amabilidad en la cotidianeidad: sonreír, ofrecer el espacio en el bus y apreciar la compañía o apoyo de sus familiares (tal fue el caso de quienes reconocieron el papel tan significativo de los abuelos en sus vidas), hecho que fomenta la intergeneracionalidad.

Las personas participantes en el curso lograron comprender que la práctica del ejercicio físico contribuye con su salud física y emocional. Asimismo, reconocieron que identificar sus fortalezas y ser agradecidos durante el transcurso de la vida les permite dar lo mejor de sí en su ámbito familiar, profesional y de relaciones sociales; que, ante los obstáculos que se presenten, sus fortalezas les pueden ayudar a superarlos más fácilmente, a aumentar su seguridad, a tener el control, a reconocer cuándo se necesita ayuda y a estar mejor consigo mismos.

Esta experiencia fue un espacio vital para la promoción de la salud y el desarrollo de la autoconciencia, además de que los alumnos aprendieron a buscar nuevos campos de aplicación de sus fortalezas y vieron la importancia de usar muchas de ellas, así como la pertinencia de empezar a ponerlas en práctica, si no lo habían hecho todavía.

Algunas frases que ejemplifican los aprendizajes son:

- “...es algo extraño... este tipo de trabajos nos hacen sentar cabeza, y nos pone a reflexionar acerca de nuestra vida...".

- "Agradezco a Dios por darme la oportunidad de perdonar".

- "No fui a pasear a ningún lugar, entonces me quedó dinero libre, por dicha" (lo que revela la interpretación positiva de los eventos).

- $\quad$ "Hoy leyendo todo lo anterior, me doy cuenta del valor de agradecer por aquellas pequeñas grandes cosas".

\section{Retos y aprendizajes desde el curso Ambiente y salud}

El curso Ambiente y salud es un curso analítico y participativo que tiene como objetivo concientizar a la población estudiantil sobre la salud como elemento clave para alcanzar tanto el bienestar como el desarrollo individual y colectivo. Hace énfasis en la importancia de adoptar prácticas de vida respetuosas con el medio y 
sobre la necesidad de desarrollar un estilo de vida que contribuya a ese estado de completo bienestar biopsicosocial conocido como salud (OMS, 2014).

Tomando en cuenta la temática del curso, las IPP representaron herramientas innovadoras y valiosas para cumplir sus objetivos. La propuesta se enmarcó en uno de los objetivos específicos: Promover la reflexión sobre el bienestar integral del ser humano. Desde el curso, se analiza la salud como requisito para lograr el bienestar del individuo, la cual está ligada a la edad de las personas, la etapa de la vida en que se encuentran, así como a las condiciones en las que se han desarrollado. En el caso de estudiantes universitarios, su etapa representa un momento de cambio y adaptación, que exige tomar decisiones, plantear y replantear metas, reconocerse a sí mismo y asumir responsabilidades en torno al presente y al futuro. Es por esto que, en la construcción de ese individuo que se quiere ser, es necesario que el joven tenga herramientas, las cuales le permitan conservar su salud y disfrutar una vida larga y de calidad.

Aunque el curso Ambiente y salud busca dar herramientas a los estudiantes para alcanzar una mejor salud, por razones varias (calendario universitario, poca capacitación de la docente, programa aprobado previamente, entre otras), no se habían incluido actividades destinadas a mejorar la salud mental y física del estudiantado en el programa. La participación de los estudiantes en el componente de psicología positiva y de ejercicio físico representó una oportunidad para incluir temas afines al curso, de una manera innovadora. Este proceso tuvo retos tanto para los estudiantes como para la profesora encargada, que obligaron a la reorganización del curso. La tabla 1 presenta los retos que acarreó el incluir la intervención en el programa de curso.

\section{Tabla 1}

Retos de la intervención en psicología positiva en el curso Ambiente y salud, 2015

\section{Profesora}

Estudiantes

Reacomodo del cronograma del Participar en sesiones de clase que curso, para poder incluir las sesiones los obligaban, de alguna forma, a en las semanas de clase establecidas. "conversar con su yo íntimo" y a compartirlo con la clase.

Motivar a los estudiantes a expresar sus ideas "introspectivas".
Las sesiones desarrollaron temas muy personales con los que no esperaban toparse e $\mathrm{n}$ un aula universitaria 
Profesora

Convencer a los estudiantes a involucrarse en actividades físicas en la clase.

Estudiantes

Participar en actividades físicas cuando muchos no tenían este aspecto incorporado en su cotidianidad y estas ponían en evidencia sus muchas o pocas cualidades físicas.

Asumir una temática de clase en la que no se es especialista, lo que implicó invertir tiempo extra en capacitación y en preparación de las lecciones.

El desarrollo de la propuesta fue muy valioso para los estudiantes y la docente, porque permitió incluir temas afines no explorados y claves para la etapa de la vida en que se encuentran los estudiantes. De ellos, los que recibieron las intervenciones combinadas de ejercicio físico y psicología positiva externaron una reducción en la frecuencia con la que experimentan emociones negativas (tristeza, ansiedad y enojo). Este efecto puede contribuir a la mejora de la vivencia de la vida universitaria y ser, a su vez, un factor protector ante riesgos asociados con el malestar psicológico en la población estudiada.

Como se indicó, un componente que estuvo presente en los cursos, además de la psicología positiva, fue el movimiento humano del que seguidamente se darán algunos aspectos que resaltaron. También, se incluyen retos y aprendizajes desde el rol de la psicología.

\section{Retos y aprendizajes desde el rol de la psicología y el movimiento humano}

Este proceso de investigación e intervención se planteó como una propuesta innovadora, que pretendió impactar positivamente en el bienestar de estudiantes universitarios, proporcionando espacios para la creación y el fortalecimiento de capacidades desde los aportes de la psicología y las ciencias del movimiento humano. Para que este planteamiento se pudiera materializar, resultó indispensable la coordinación con las autoridades del CEG, así como la disposición del profesorado a cargo de los cursos. 
En una primera etapa, se trabajó únicamente con el personal académico, en sesiones con metodología participativa, para la sensibilización y formación en aspectos teóricos y metodológicos de la psicología positiva en general y de las intervenciones específicas. En este primer momento, también fue necesario que la profesional en psicología realizara un esfuerzo para la aproximación y comprensión de las temáticas de cada uno de los cursos, de manera que pudiera brindarse realimentación con respecto a posibles formas en que las temáticas y metodologías (de los cursos y de las intervenciones tanto de psicología positiva como de ejercicio físico) pudieran interactuar de manera coherente y provechosa para el estudiantado. A partir de este trabajo, se concretó un protocolo por seguir para las académicas, con miras al desarrollo de las IPP en el aula.

El diseño de un protocolo para las sesiones de ejercicio físico que se adaptara al tiempo y los espacios físicos disponibles fue posible gracias al trabajo conjunto con un profesional en educación física. La profesional en psicología, quien a su vez es docente en ámbitos académicos que integran la psicología y las ciencias del movimiento humano, precisó algunos requerimientos en cuanto a duración, intensidad y frecuencia de las actividades por realizar.

Durante las semanas de ejecución de las intervenciones, se llevó a cabo un trabajo de seguimiento conjunto entre el personal académico y la psicóloga, de manera que se garantizara el cumplimiento del protocolo establecido. Asimismo, la psicóloga brindó realimentación sobre las asignaciones realizadas por los estudiantes, facilitando elementos que pudieran contribuir a las discusiones en clase.

Debido que este proceso se desarrolló en el ambiente cotidiano de las aulas universitarias, existen diversas variables sobre las que resulta difícil ejercer control y que pueden influir en los resultados. Si bien se procuró medir algunas de ellas (como el nivel de actividad física, el socioeconómico y la participación en procesos terapéuticos), otros como los eventos positivos o negativos que pudieran ocurrir en la vida de los participantes en el periodo de la intervención (logros personales, pérdidas, cambios en el núcleo familiar o social cercano) no fueron tomados en cuenta.

El diseño, la preparación y la ejecución de este proyecto requirió un esfuerzo importante en cuanto a coordinaciones, logística y seguimiento; los resultados demuestran que es factible integrar diversas disciplinas en un proceso con miras a la promoción de la salud y el bienestar del estudiantado, desde las mismas aulas universitarias. 


\section{Logros y perspectivas a futuro}

Por último, en este apartado, se presentan logros y perspectivas a futuro, acerca de la experiencia de integrar intervenciones de psicología positiva y ejercicio físico en diversos cursos de Estudios Generales. Dicha experiencia generó posibilidades para que las diferentes disciplinas trabajaran de manera estrecha en un plan común para la praxis académica en la docencia e investigación, en un proceso que es coherente con el enfoque de los Estudios Generales, descrito por Baraona y Mata (2015) como pedagogía multidisciplinaria que se enrumba hacia la interdisciplina.

La percepción de estudiantes, académicas y la profesional en psicología en cuanto a los retos y aprendizajes de esta experiencia (reseñados en los apartados previos) da cuenta de cómo se posibilitaron espacios para la promoción del bienestar psicológico de estudiantes universitarios, mediante las intervenciones propuestas, así como para el desarrollo y fortalecimiento de capacidades en el personal académico del Centro de Estudios Generales, con miras a gestionar intervenciones de psicología positiva. A partir de lo vivenciado, surgen perspectivas por considerar para la continuación de esta línea de trabajo. Por una parte, el reto a futuro que enfrentan los académicos sería el de generar capacidades en sí mismos y en sus estudiantes para progresar hacia una educación más equitativa y respetuosa de la diversidad, tanto a través de los procesos de aprendizaje como de la articulación de diferentes técnicas y estrategias para desarrollar las actividades de los diferentes cursos, en conjunto con la psicología positiva y el ejercicio físico.

Desde el Taller de expresión literaria se identificó que es posible que los jóvenes universitarios logren aprendizajes significativos, mediante la promoción de la lectura y la escritura creativa, la adquisición de nuevos conocimientos, el rescate de valores, la creación de espacios para la socialización y la apreciación de obras literarias, entre otros. Este tipo de trabajos conjuntos entre la psicología positiva y la literatura se convierten en una oportunidad para que los estudiantes puedan enfrentar retos y desafíos de manera creativa e innovadora, permitiendo nuevas percepciones, al otorgar novedosos significados a sus proyectos de vida.

Se visualiza que resultaría beneficioso que se continúe incorporando las intervenciones de psicología positiva y el fomento del ejercicio físico en el curso La imagen de la persona adulta mayor en los diferentes textos cuando se desarrolle el tema de estilos de vida saludables y longevidad en el curso de vida.

Resulta relevante reseñar que, a partir de esta experiencia en el programa de Ambiente y salud, se incluyó una sesión que presenta algunas generalidades de 
psicología positiva y se desarrolla el tema de "mis herramientas" tal cual se hizo en la intervención en el II ciclo 2015. El objetivo de la clase es que los estudiantes reconozcan sus herramientas emocionales (fortalezas personales) para poder enfrentar, de la mejor manera, las diversas situaciones de vida que enfrentan. Lo anterior es una muestra de la continuidad del proceso, gracias a la generación y el fortalecimiento de capacidades en el personal académico.

Tomando en cuenta lo anterior, se considera pertinente y relevante fomentar el desarrollo de capacitaciones en los docentes del CEG, acera de las temáticas de psicología positiva y ejercicio físico, con el fin de que desde sus cursos contribuyan con el desarrollo integral de los estudiantes.

A la luz de los resultados de las intervenciones y la corriente del nuevo humanismo que se desarrolla en el CEG, se podría plantear un curso interdisciplinario que trabaje, desde una visión holística, el componente de ejercicio físico.

Con base en la perspectiva del proceso de investigación, resultaría interesante que en experiencias venideras se utilicen diseños de investigación longitudinales que abarquen el periodo correspondiente a la vida universitaria. Si se llevan a cabo intervenciones durante el primer año de estudios, un enfoque de este tipo posibilitaría monitorear los efectos a largo plazo. También podría analizarse algunas variables relevantes para el ámbito de la educación superior, como rendimiento académico, deserción y rezago tanto en los planes de estudio como en los estilos de vida.

Se considera que esta experiencia aporta al desarrollo de nuevas formas de conceptualizar y ejercer la praxis académica en los Estudios Generales. Permite aproximarse a lo que Pawelski (2016) y Pawelski y Moores (2012) denominan "humanidades positivas", desde las cuales se enfatiza la importancia de contribuir a una comprensión más profunda del bienestar, así como al desarrollo de formas más efectivas de promoverlo en las aulas universitarias. Este enfoque de las humanidades positivas pareciera coincidir con las propuestas del nuevo humanismo, desde el cual se abraza toda aquella doctrina que proponga el perfeccionamiento de la vida colectiva e individual humana (Baraona y Mata, 2015), y el relato de las experiencias reseñadas evidencia que las aspiraciones de ambas visiones pueden materializarse, al integrar esfuerzos de diferentes disciplinas. En este caso particular, los logros se reflejan en la promoción del bienestar psicológico de estudiantes universitarios y tanto el desarrollo como el fortalecimiento de capacidades en el personal académico del Centro de Estudios Generales. 


\section{Referencias}

Baraona, M. (2013). Para un humanismo del siglo XXI: desafíos y propuestas. Revista Nuevo Humanismo, 1(1)

Baraona, M. y Mata, E. (2015). Los Estudios Generales como pedagogía multidisciplinaria en vías a la interdisciplina. Revista Nuevo Humanismo, 3(1).

Baraona, M. y Mora, J. (2017). Hacia una epistemología del Nuevo Humanismo. Costa Rica: Editorial EUNED.

Barrantes, K. (2017). Efecto de intervenciones de psicología positiva y ejercicio en el bienestar y malestar psicológico de estudiantes universitarios. Doctorado en Ciencias del Movimiento Humano, Heredia: Sistema de Estudios de Posgrado, Universidad Nacional-Universidad de Costa Rica.

Bauman, A., Schoeppe, S., Lewicka, M., Armstrong, T., Candeias, V. y Richards, J. (2008). Review of best practice in interventions to promote physical activity in developing countries. Recuperado de: http://www.who.int/dietphysicalactivity/ bestpracticePA2008.pdf

Bolier, L., Haverman, M., Westerhof, G. J., Riper, H., Smit, F. y Bohlmeijer, E. (2013). Positive psychology interventions: a meta-analysis of randomized controlled studies. BMC Public Health, 13, 119, 1-20 Doi: 10.1186/1471-2458-13-11

Buettner, D. (2008). The Blue Zones: Lessons for living longer from the people who've lived the longest. Washington, D.C.: National Geographic.

Comunicación personal de una estudiante. Curso Taller de expresión literaria: cuento y poesía, 31 de agosto, 2015. Heredia, Universidad Nacional de Costa Rica.

Emmons, R. A. y McCullough, M. E. (2003). Counting blessings versus burdens: An experimental investigation of gratitude and subjective well-being in daily life. Journal of Personality and Social Psychology, 84(2), 377-389. Doi: 10.1037/0022-3514.84.2.377

Flay, B., Biglan, A., Boruch, R., Castro, F., Gottfredson, D., Kellam, S. y Ji, P. (2005). Standards of evidence: criteria for efficacy, effectiveness and dissemination. Prevention Science, 6 (3), 151-175. Doi: 10.1007/s11121-005-5553-y

Hervás, G., Sánchez, A. y Vázquez, C. (2008). Intervenciones psicológicas para la promoción del bienestar. En G. C. Vázquez y Hervás (ed.), Psicología Positiva Aplicada (2. a ed., pp. 41-74). Bilbao: Desclée de Brouwer.

Hoehner, C., Soares, J., Parr, D., Ribeiro, I., Joshu, C., Pratt, M. y Brownson, R. (2008). Physical activity interventions in Latin America: a systematic review. 
American Journal of Preventive Medicine, 34(3), 224-233. Doi: 10.1016/j. amepre.2007.11.016

Hone, L., Jarden, A. y Schofield, G. (2014). An evaluation of positive psychology intervention effectiveness trials using the re-aim framework: A practice-friendly review. Journal of Positive Psychology, 10(4), 1-20. Doi: 10.1080/17439760.2014.965267

Huijg, J., Zouwe, N., Crone, M., Verheijden, M., Middelkoop, B. y Gebhardt, W. (2015). Factors influencing the introduction of physical activity interventions in primary health care: A qualitative study. International Journal of Behavioral Medicine, 22(3), 404-414. Doi: 10.1007/s12529-014-9411-9

Iacub, R. (2007). La trascendencia y el sentido de la vida. Recuperado de http://www. ricardoiacub.com.ar/la-trascendencia-y-el-sentido-de-la-vida/

King, L. (2001). The health benefits of writing about life goals. Personality \& Social Psychology Bulletin, 27(7), 798. Doi: 10.1177/0146167201277003

Laine, J., Kuvaja-Köllner, V., Pietilä, E., Koivuneva, M., Valtonen, H. y Kankaanpää, E. (2014). Cost-effectiveness of population-level physical activity interventions: A systematic review. American Journal of Health Promotion, 29(2), 71-80. Doi: 10.4278/ajhp.131210-LIT-622

Layous, K., Nelson, S. y Lyubomirsky, S. (2013). What is the optimal way to deliver a positive activity intervention? The case of writing about one's best possible selves. Journal of Happiness Studies, 14(2), 635-654. Doi: 10.1007/s10902-012-9346-2

Miller, E. y Chung, H. (2009). A literature review of studies of depression and treatment outcomes among U.S. College students since 1990. Psychiatric Services, 60(9), 1257-1260. Doi: 10.1176/appi.ps.60.9.1257

Organización Mundial de la Salud. (2014). Documentos Básicos Edición 48. Recuperado de http://apps.who.int/gb/bd/PDF/bd48/basic-documents-48th-edition-sp. pdf\#page $=7$

Park, N. y Peterson, C. (2009a). Achieving and sustaining a good life. Perspectives on Psychological Science, 4(4), 422-428. Doi:10.1111/j.1745-6924.2009.01149.x

Park, N. y Peterson, C. (2009b). Character strengths: Research and practice. Journal of College \& Character, 10(4), 1-10. Doi: 10.2202/1940-1639.1042

Park, N., Peterson, C. y Seligman, M. E. P. (2004). Strengths of character and well-being. Journal of Social and Clinical Psychology, 23(5), 603-619. Doi: 10.1521/ jscp.23.5.603.50748 
Parks, A. y Biswas-Diener, R. (2013). Positive interventions: Past, present, and future. En T. Kashdan y J. Ciarrochi (eds.), Mindfulness, Acceptance, and Positive Psychology: The Seven Foundations of Well-Being, 140-165. Oakland, CA: Context Press.

Pawelski, J. (2016). What is the role of the Humanities in positive education? Blog post. Recuperado de http://www.ipositive-education.net/ what-is-the-role-of-the-humanities-in-positive-education/

Pawelski, J. y Moores, D. (2012). The eudaimonic turn: Well-being in literary studies. Lanham: Rowman \& Littlefield.

Peterson, C. (2006). A primer in positive psychology. New York: Oxford University Press.

Peterson, C., Ruch, W., Beermann, U., Park, N. y Seligman, M. (2007). Strengths of character, orientations to happiness, and life satisfaction. Journal of Positive Psychology, 2(3), 149-156. Doi: 10.1080/17439760701228938

Schueller, S., Kashdan, T. y Parks, A. (2014). Synthesizing positive psychological interventions: Suggestions for conducting and interpreting meta-analyses. International Journal of Wellbeing, 4(1), 91-98. Doi: 10.5502/ijw.v4i1.5

Seligman, M. y Csikszentmihalyi, M. (2000). Positive psychology. An introduction. American Psychologist, 55(1), 5-14. Doi: 10.1037/0003-066X.55.1.5

Seligman., Rashid, T. y Parks, A. (2006). Positive psychotherapy. American Psychologist, 61(8), 774-788.

Seligman, M., Steen, T., Park, N. y Peterson, C. (2005). Positive psychology progress: Empirical validation of interventions. American Psychologist, 60(5), 410-421. Doi: 10.1037/0003-066x.60.5.410

Sheldon, K. y King, L. (2001). Why positive psychology is necessary. American Psychologist, 56(3), 216. Doi: 10.1037/0003-066X.56.3.216

Sheldon, K. y Lyubomirsky, S. (2006). How to increase and sustain positive emotion: The effects of expressing gratitude and visualizing best possible selves. Journal of Positive Psychology, 1(2), 73-82. Doi: 10.1080/17439760500510676

Sin, N. y Lyubomirsky, S. (2009). Enhancing well-being and alleviating depressive symptoms with positive psychology interventions: a practice-friendly meta-analysis. Journal of Clinical Psychology, 65(5), 467-487. Doi: 10.1002/jclp.20593

Wood, A., Froh, J. y Geraghty, A. (2010). Gratitude and well-being: A review and theoretical integration. Clinical Psychology Review, 30(7), 890-905. Doi: 10.1016/j. cpr.2010.03.005 